IRA-International Journal of Technology \& Engineering

ISSN 2455-4480; Vol.04, Issue 01 (2016)

Institute of Research Advances

http://research-advances.org/index.php/IRAJTE

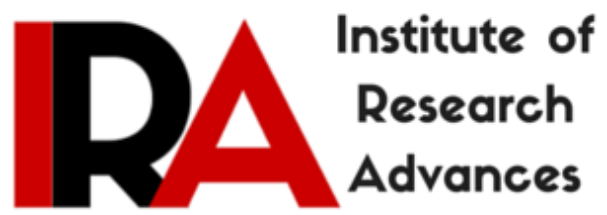

\title{
Laser Speckle Interferometer for Deformation Measurement based on Fast Fourier Transform method
}

\section{R. Balamurugan \\ Department of Physics, Kumaraguru College of Technology, Coimbatore- 641049, India.}

DOI: http://dx.doi.org/10.21013/jte.v4.n1.p5

\section{How to cite this paper:}

Balamurugan, R. (2016). Laser Speckle Interferometer for Deformation Measurement based on Fast Fourier Transform method. IRA-International Journal of Technology \& Engineering (ISSN 2455-4480), 4(1). doi:http://dx.doi.org/10.21013/jte.v4.n1.p5

(C) Institute of Research Advances

\section{(cc) EY-NC}

This works is licensed under a Creative Commons Attribution-Non Commercial 4.0 International License subject to proper citation to the publication source of the work.

Disclaimer: The scholarly papers as reviewed and published by the Institute of Research Advances (IRA) are the views and opinions of their respective authors and are not the views or opinions of the IRA. The IRA disclaims of any harm or loss caused due to the published content to any party. 


\begin{abstract}
In this paper, Laser speckle image processing based on Fast Fourier Transform method for displacement measurement is presented. Capturing Laser speckle images before displacement and after displacement of the specimen is the technique involved in this measurement. The cross correlation is computed by two 2Dimensional Fast Fourier Transforms (one direct and the other is inverse) and a complex multiplication. This is a non-contact, flexible measurement with high accuracy and simple operation. The results are verified by conventional gauge methods.
\end{abstract}

Keywords: laser speckle, image correlation, displacement

\title{
Introduction
}

The optical principle, governing the laser speckle photography and speckle interferometry is well established [1-3]. Speckle interferometry is used to measure the deformation/displacement of micro electromechanical systems [4], medical studies on bone dynamics to quality inspection of various products [5] and the measurement of the refractive index of a liquid in a cell was reported [6]. Laser speckles is a phenomenon that is experienced if an optically rough surface is illuminated by a highly coherent laser source. They are formed by the interference of the random phased but coherent wavelets, emanating from different microscopic elements of the specimen's surface and cause a randomly looking pattern like fingerprint[7]. We propose a simplified Digital speckle correlation method for the experimental evaluation of deformation/displacement and results are verified by conventional mechanical gauge methods.

\section{Experimental setup}

The optical setup for speckle interferometry is based on the Michelson interferometer. The specimen object is a rectangular aluminum plate with a size of $220 \mathrm{~mm}$ and $165 \mathrm{~mm}$. A 5-mW laser diode is a coherent source having wavelength $680 \mathrm{~nm}$. A lens is used to increase the laser divergence to illuminate the target surface suitably. The beam splitter divides the laser into two parts and each of these beams illuminates different surfaces. An optical system with a converging lens is used to collect the diffused light and project the image onto a screen. Double exposure method is adopted here. Before the deformation and after deformation of the object is recorded.

\section{Fourier Transform Method}

Fourier type methods are preferred than correlation when we require computational speedup and when the images get corrupted by frequency dependent noise[9]. That is a treatment in frequency domain. Fourier Shift Theorem is used in phase-correlation methods. Reddy and Chatterji [10] computes the power spectrum of the images from its cross-correlation and it looks for the peak in its inverse to be measured. The two images acquired before and after deformation is sub divided into set of sub images. The correlated function is calculated for each pair of images and the respective displacement is derived from the position of maximum. The cross correlation is computed with two 2D FFTs (one direct and one inverse) and a complex multiplication. The size of the image decides the number of FFT slices to be used. Therefore the displacement is sampled at the center of the sub images. The size of the sub images are chosen like $32 \times 32$ or $64 \times 64$ determines the spatial resolution and the accuracy of measurement. The finite $\mathrm{B}$ of the correlation window limits the spatial resolution and the spectral contents in the displacement field to frequencies of less than 1/B. [11]

\section{Result and analysis}

The desired information retrieved by performing a Fourier-based demodulation. First the image is Fast Fourier transformed (FFT). Then, filtering in the frequency plane, we select a side lobe and shift it into the origin (filtering). Inverse FFT (IFFT) yields a complex image from which obtaining the phase (arctan). This phase is wrapped in the range $-\pi: \pi$. Figure.(1) illustrated FFT speckle images.The Matlab 
output shows Figure.(2) gives the information of displacement measurement and fairly matched with the mechanical tools (Gauge) measurements. The proposed architecture will reduce the correlation computation by more than two orders of magnitudes with respect to software implementations without loss of accuracy.

\section{Conclusion}

In this paper, a simple electronic speckle photography experiment to detect small deformation of an Aluminum plate has been done. The setup is of simple construction and only a few optical elements are needed: a laser, a camera, and a light scattering plate. We propose a simplified Digital speckle correlation method for the experimental evaluation of deformation/displacement and results are verified by conventional mechanical gauge methods.

\section{Acknowledgements}

The authors would like to thank the management of Kumaraguru College of Technology for the continuous support for this work.

\section{References}

[1] Yamaguchi,I“A laser-speckle strain gauge”, J. Phys.E: Sci. Instrum.,1981,14,1270-273.

[2] Goodman, J. W. "Introduction to Fourier Optics", McGraw-Hill, New York, 1968.

[3] Sjodahl, M. "A whole field speckle strain sensor", In Proc. SPIE Conference on Optical Engineering. Yokohama, 1999.

[4] K. Erf "Speckle Metrology", (Academic, New York, 1978).

[5] P. Aswendt, C. D. Schmidt, D. Zielke, and S. Schubert, "ESPI solution for non contacting MEMS-on-wafer testing,’ Opt. Lasers Eng. 40, 501-515,(2003)

[6] P. K. Buah-Bassuah, F. Francini, and G. Molesini, "Measurement of refractive index by double-exposure speckle pattern recording,'’Am. J.Phys. 57, 366-370, 1989.

[7] Ganesan A.R., "Proceedings of the National Seminar \& Exhibition on Non-Destructive Evaluation NDE 2009", December 10-12, 2009

[8] Sirohi, R.S., Tay, C.J., Shang, H.M., Boo, W.P.: "Nondestructive assessment of thinning of plates using digital shearography." Opt.Eng. 38(9), 1582-1585 (1999)

[9] T. Mahalakshmi, R. Muthaiah and P. Swaminathan, "Review Article: An Overview of Template Matching Technique in Image Processing", Research Journal of Applied Sciences, Engineering and Technology 4(24): 5469-5473, 2012

[10] Reddy, B.S. and B.N. Chatterji, 1996. An FFT-based technique for translation, rotation and scale-invariant image registration. IEEE T. Image Proc., 5(8): 1266- 1271.

[11].Balamurugan Rajamanickam, "Application of Fast Fourier Transform (FFT) in Laser Speckle Image Pattern Correlation Technique for the Metrological Measurement", IEEE Fourth International conference on Advanced computing 10.1109/ICoAC.2012.6416803, p.1-4, 2012. 
Figure.1
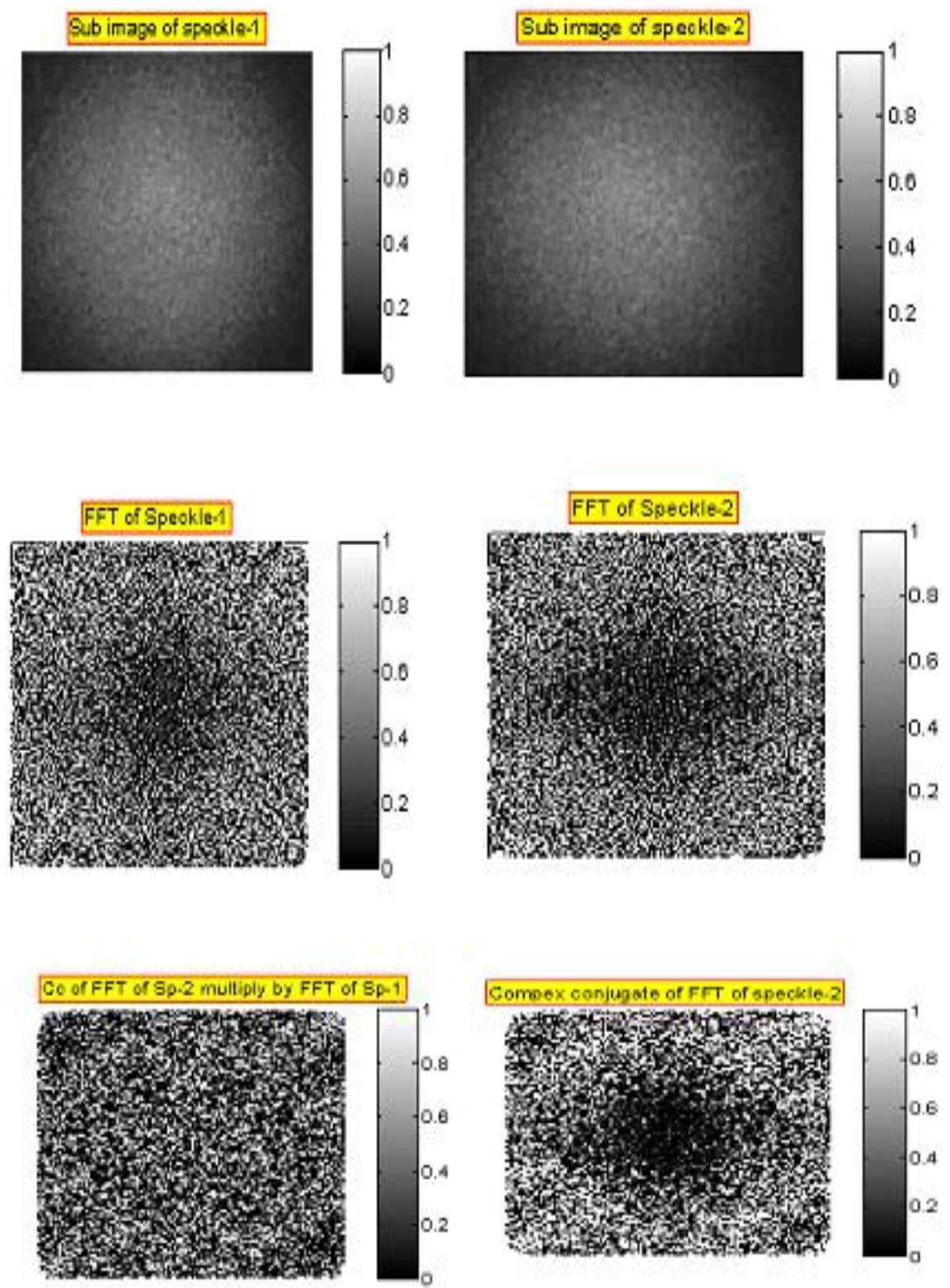

Figure.(1) Illustration of Processing of sub images 
Figure.2

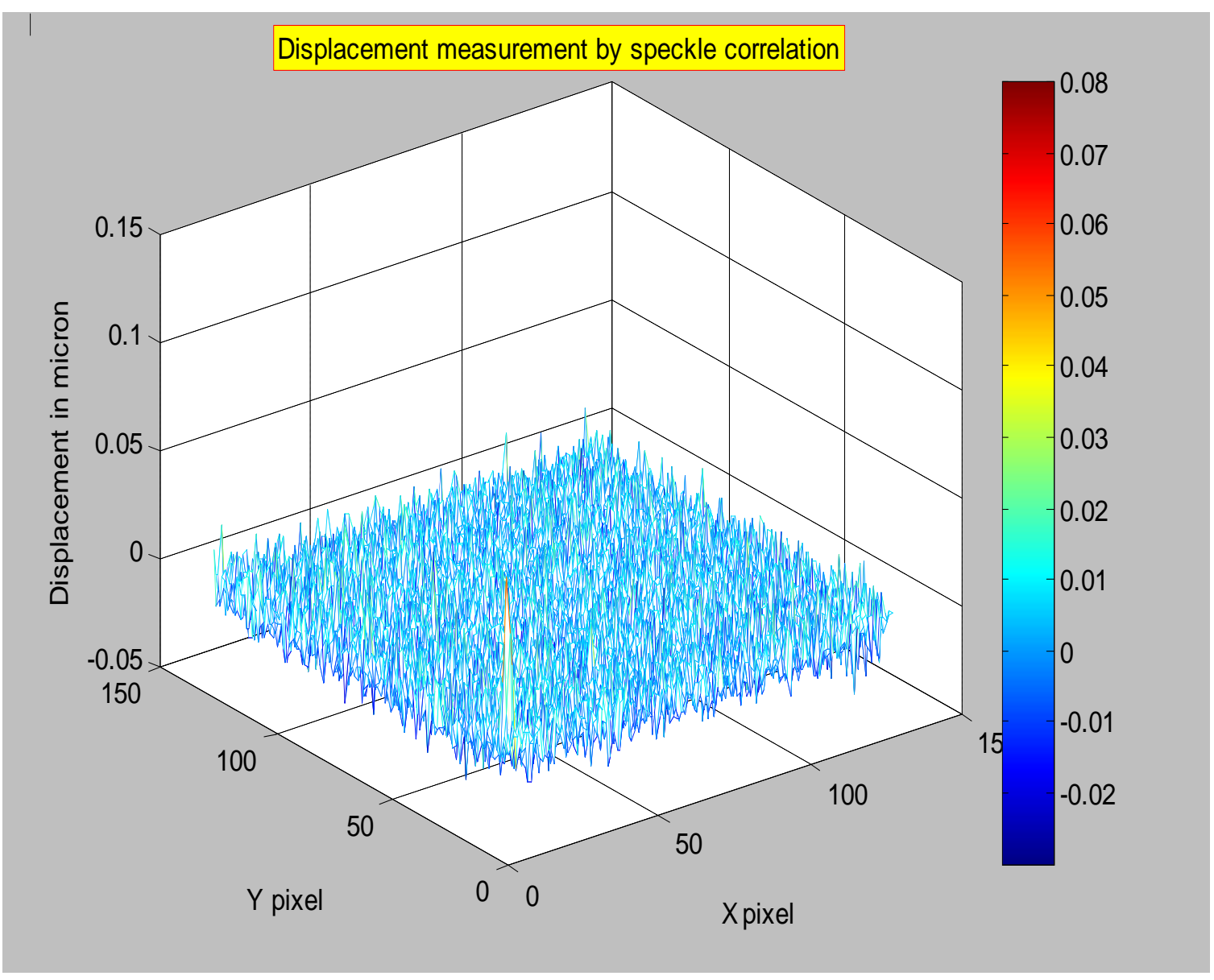

Figure.(2). Displacement measurement result (0.08 micron) by FFT method 